\title{
Prevalência e perfil dos usuários de álcool de população adulta em cidade do sul do Brasil
}

\author{
Prevalence and alcohol user profile in adult population \\ in a South Brazilian city
}

M arcelo Carlos Bortoluzzi ${ }^{1}$

Jefferson Traebert ${ }^{2}$

Alessandro Loguercio ${ }^{3}$

Ruth Terezinha Kehrig ${ }^{4}$

\footnotetext{
${ }^{1}$ Faculdadede Odontologia. Universidade do Oestede Santa Catarina. Av. Getúlio Vargas 2125. Bairro Flor da Serra. 89600-000 Joaçaba

SC. mbortoluzzi@gmail.com

${ }^{2}$ Programa de M estrado em

Ciências da Saúde,

Universidade do Sul de

Santa Catarina.

${ }^{3}$ Programa de M estrado em Odontologia, Universidade

Estadual de Ponta Grossa.

${ }^{4}$ UniversidadeFederal do

M ato Grosso.
}

Abstract This is an observational cross-sectional survey which included 707 individualsfrom a south Brazilian city (J oaçaba, in Santa Catarina State) aiming to know the alcohol user prevalence as well as the profile of the user. The results showed that $45.5 \%$ (322) of that population consume alcohol on regular basisand had used it at least oncein the last month. The regular alcohol consumption occurs predominantly on males $(p<0.001)$, in people under 39 years old ( $p=0.007$ ), occurring predominantly with ones working comparing to the ones not working, $(p<0.001)$, have more than 8 years of education $(p<0.001)$ and with income greater than 1738.00 reais - Brazilian currency $(p<0.001)$. The regular alcohol consumption was greater on those who classified his health status as regular, good or very good $(p<0.006)$, also this relation happen to those people who haven't been under hospital internment in the last year ( $p$ $<0.013)$. The depression levels scored by the Beck Depression Inventory (BDI) showed low levels to those who regularly consume alcohol ( $p<0.047$ ). Key words Alcohol, Prevalence, User profile, BAI, BDI
Resumo Esteéum estudo do tipo observacional e transversal envolvendo 707 indivíduos do município de Joaçaba (SC), que visa conhecer a prevalência de usuários regulares de álcool, bem como seu perfil. Osresultados mostram que 45,5\% (322) fazem uso de álcool de forma regular e consumiram álcool ao menos uma vez no último mês. 0 consumo regular de álcool ocorre predominantementeno sexo masculino $(p<0,001)$, entrepessoas abaixo dos 39 anos de idade ( $p=0,007)$, ocorre predominantemente naqueles que trabalham em relação aqueles que não estão trabalhando ( $p$ $<0,001)$, entre os que têm mais de oito anos de estudo ( $p<0,001$ ) ecom renda superior a 1.738,00 reais $(p<0,001)$. 0 consumo regular de álcool declarado foi maior na população que considerou sua saúde como regular, boa ou muito boa ( $p$ $<0,006$ ), bem como esta relação surge naquelas pessoas que não estiveram internadas no último ano $(p<0,013)$. Os níveis de depressão medidos pelo Inventário deBeck para D epressão (BDI) mostraram menores índices de depressão para aqueles que consomem regularmente álcool $(p<0,047)$. Palavras-chave Álcool, Prevalência, Perfil de usuários, BAI, BDI 
Introdução

A Organização Mundial da Saúde (OM S) estima que atualmente existam dois bilhões de pessoas em todos os continentes que consomem bebidas al coólicas e cerca de 76,3 milhões convivem com diagnóstico de desordens relacionadas ao consumo destas bebidas, o que, por esta razão, traz uma carga social e econômica considerável sob a perspectiva da saúde pública. Ainda, segundo a OM S, a população brasileira encontra-se entre osmaiores consumidores deálcool, com estimativa de consumo anual de aproximadamentenove litros deálcool absoluto entre residentes maiores de quinze anos de idade.

Globalmente, o álcool provoca 3,2\% de todas as mortes ou, cerca de 1,8 milhões de mortes anuais e, ainda, cerca de $4 \%$ das doenças estão relacionadas ao seu uso. Do número total de mortes atribuídas ao álcool, 32\% são resultantes deinjúrias não intencionais, ou seja, acidentes de trânsito, afogamentos, queimaduras, quedas e outras².

Apesar do grande potencial para provocar mortalidade e morbidade, o consumo de álcool continua crescendo em países em desenvolvimento, enquanto permaneceu estável ou diminuiu na Europa Ocidental e América do N orte entre os anos de 1961-19973. Contudo, um relatório de vigilância também indica um aumento de 0,4\% consumo per capita de álcool nos Estados Unidos da América no ano de 2005 em relação ao ano de $2004^{4}$.

Cabe ressaltar que é meta na constituição de políticas do M inistério da Saúde para os próximos anos a apreensão do fenômeno contemporâneo do uso abusivo/dependência em álcool e outras drogas de modo integrado 5 . Este é um estudo de base populacional, transversal quevisa conhecer a prevalência de usuários regulares de álcool, bem como seu perfil.

\section{M ateriais emétodos}

Foi realizado um estudo do tipo observacional e transversal envolvendo uma amostra representativa da população adulta compreendida entre 20 a 59 anos de idade do município de Joaçaba (SC) no ano de 2005. Este estudo éparte de uma pesquisa maior denominada Projeto Saúde Joaçaba ${ }^{6,7}$, que visa conhecer diversos aspectos relacionados à saúde dos cidadãos do município.

Para a determinação do tamanho da amostra com intervalo de confiança de $95 \%$, foram adota- dos os seguintes parâmetros: proporção de situações autorreferidas de saúde desconhecida ( $P$ igual a 50\%) e margem de erro de $4 \%$. 0 tamanho da amostra obtido foi de 575 participantes. Considerando-se um fator de correção de 1,2 em função do desenho do estudo, este valor acresceu para 690 indivíduos. Estimando-se perdas, recusas e potenciais fatores de confusão de $20 \%$, 0 número final da amostra foi de 828 indivíduos.

A seleção da amostra foi realizada pelo método de amostragem por conglomerados ${ }^{8}$, utilizando como unidade de referência para localização da população os setores censitários. Todos os setores censitários existentes no município de Joaçaba $(n=25)$ participaram do estudo eaamostra foi composta por 33 indivíduos de cada setor. Através de mapas cartográficos, foram identificados e numerados todos os quarteirões dos setores. Em cada setor, foi sorteado um quarteirão e, neste, uma esquina foi selecionada para o início do estudo. Foram percorridos os doze primeiros domicílios, no sentido horário da esquina sorteada, necessários para a obten ção do número de indivíduos estimados para compor a amostra em cada um dos 25 setores censitários. Todos os indivíduos que residiam no domicílio sorteado eque pertenciam à faixa de 20 a 59 anos foram entrevistados. Ao total, foram visitados 345 domicílios.

0 instrumento de pesquisa global foi composto por um questionário que continha questões que visavam (1) identificar aspectos demográficos esocioeconômicos, (2) identificar condições relacionadas aos processos ecológicos do ambiente residencial ede trabalho, (3) identificar a ocorrência de fatores de risco selecionados à saúde derivados de hábitos, condutas e estilo de vida, (4) estimar a prevalência de afastamento do trabalho por problemas de saúde, (5) estimar a prevalência de problemas desaúdemental einsatisfação com aparência corporal, (6) estimar a prevalência de problemas respiratóriose, (7) descrever as necessidades autorreferidas de serviços de saúde. 0 questionário, portanto, foi formulado de forma a atender os objetivos específicos da pesquisa, o que incluía 193 questões elaboradas de acordo com a literatura vigente ${ }^{6}$. Além deste instrumento, instrumentos validados foram utilizados para avaliar condições específicas como os níveis de ansiedade, que foram mensurados pelo Inventário Beck de Ansiedade (BAI), ou os níveis de depressão, que foram mensurados pelo Inventário de Beck para Depressão (BDI)9.

O BAI consta de uma lista de 21 sintomas com quatro alternativas cada um, em ordem cres- 
cente do nível de ansiedade. A classificação brasileira propõe como resultados os níveis de 0 a 9 como mínimo, de 10 a 16 como leve e segue até uma pontuação máxima de 63 numa escala de moderada a grave. Para fins de análise, a amostra foi dicotomizada estabelecendo que de 0 a 16 a ansiedade fosse caracterizada como mínima e/ ou leve e, acima deste, como moderada a grave. O BDI mede as manifestações comportamentais da depressão e a soma dos escores identifica o nível de depressão. A classificação brasileira propõe que de 0 a 11 o nível de depressão seja mínimo e de 12 a 19 o nível da depressão élevee segue uma pontuação até 63 , que representa índices de depressão também crescentes. N o presente estudo, a amostra foi dicotomizada estabel ecendo que de 0 a 19 fosse caracterizada como mínima e/ou leve e acima deste ponto foi considerada como moderada a grave. A dicotomização estabelecida para o BAI eBDI seguea classificação brasileira9 ${ }^{9}$.

A equipe de trabalho de coleta dos dados foi composta por sete pesquisadores e sete auxiliares de pesquisa, que passaram por um processo de capacitação para assegurar a uniformidade da aplicação do instrumento de pesquisa, de forma a minimizar variações entre as observações realizadas pelos diferentes pesquisadores integrantes do projeto. 0 trabalho de campo ocorreu nos meses de dezembro de 2005 a março de 2006.

Os resultados são descritos e analisados de forma descritiva e analítica. Os testes de associação foram realizados utilizando o programa es- tatístico SPSS 13.0 para Windows a um nível de significância dep $<0,05$.

0 este estudo foi submetido ao Comitê de Ética em Pesquisa da Universidade do Oeste de Santa Catarina (CEP UNOESC/HUST) no processo parecer $n \cong$ 251/2005.

\section{Resultados}

Do total de 828 indivíduos da amostra, foram entrevistados 707, proporcionando uma taxa de resposta de $85,4 \%$. A idademédia foi de 38,7 anos $( \pm 11,7)$, sendo que $60 \%$ da amostra foram compostos pelo sexo feminino (424). Do total da amostra, 45,5\% (322) relatam consumir álcool frequentemente. Os entrevistadosquedeclararam usar álcool frequentemente (322) afirmam ter feito uso desta substância ao menos uma vez no último mêse, desta forma, foram classificados como "consumidores regulares". Dentre os consumidores regulares, 85,1\% (274) declararam a ingestão de álcool até quatro dias no último mês. 0 percentual daqueles que declararam a ingestão deálcool mais de 25 dias no último mês foi de $0,6 \%$ (2). U so de álcool, por dias de consumo no último mês, pode ser observado na Tabela 1.

Considerando a dose de álcool consumida como uma lata de cerveja, uma taça de vinho, um drink de bebida destilada ou cocktail, em relação ao dia em que fez uso de álcool no último mês, a média foi de $2,5( \pm 2,8)$ doses/dia/usuário.

Tabela 1. Uso de álcool conforme os dias de consumo no último mês e a frequência de usuários.

\begin{tabular}{cccc}
\hline $\begin{array}{c}\text { Dias de consumo deálcool } \\
\text { no último mês (dias) }\end{array}$ & $\begin{array}{c}\text { Frequência de } \\
\text { usuários }(\mathrm{n})\end{array}$ & $\begin{array}{c}\text { Percentagem de usuários } \\
\text { do total da amostra (\%) }\end{array}$ & $\begin{array}{c}\text { Percentagem cumulativa } \\
\text { entre usuários (\%) }\end{array}$ \\
\hline 1 & 154 & 21,8 & 47,8 \\
2 & 77 & 10,9 & 71,7 \\
3 & 28 & 4,0 & 80,4 \\
4 & 15 & 2,1 & 85,1 \\
5 & 12 & 1,7 & 88,8 \\
6 & 4 & 0,6 & 90,1 \\
7 & 24 & 3,4 & 97,5 \\
8 & 3 & 0,4 & 98,4 \\
10 & 3 & 0,4 & 99,4 \\
25 & 1 & 0,1 & 99,7 \\
30 & 1 & 0,1 & 100 \\
Total parcial & 322 & $45,5 \%$ & - \\
Não consome álcool & 385 & $54,5 \%$ & - \\
Total geral & 707 & $100 \%$ & - \\
\hline
\end{tabular}


Beber consumindo um volume excessivo de álcool num curto espaço de tempo é uma prática conhecida na literatura internacional como "binge drinking", ou "beber em binge". A quantidade que define o "beber em binge" é reconhecida como cinco doses para homens e quatro doses para mulheres, em uma só ocasião. Cabe notar que, entre os consumidores regulares, "beber em binge" no último mês representou $14,9 \%$, considerando ambos os sexos (Tabela 1).

Entreos entrevistados, o consumo regular de álcool ocorreu predominantementeno sexo masculino (qui-quadrado, $p<0,001$; OR 4,28; IC 95 $\%=3,1$ a 5,9 ). Embora o consumo seja frequente em todas as idades, existeuma maior frequência de consumidores regulares de álcool entre pessoas abaixo dos 39 anos de idade (mediana) (qui-quadrado, $p=0,007$; OR 1,5; IC $95 \%=1,1$ a 2,2). 0 fato de estar trabalhando no momento da entrevista mostrou uma maior frequência de consumidores regulares de álcool em relação a não estar trabalhando (qui-quadrado, $p<0,001$; OR 1,8; IC $95 \%=1,3$ a 2,5). A proporção de usuários regulares de álcool também foi maior na população com mais de oito anos de estudo (qui-quadrado, p <0,001; OR 2,0; IC $95 \%=1,4$ a 2,7) e com renda superior a 1.738,00 reais ( $m e$ diana) (qui-quadrado, $p<0,001$; OR 2,1; IC 95 $\%=1,5$ a 2,9). Contudo, não foi observada correlação entre a frequência de dias de uso deálcool e a renda familiar total $(r=0,06 ; p=0,27$; IC $95 \%=0,05$ a 0,17 ).

Considerar sua saúde como regular, boa ou muito boa mostrou uma relação significante na população que consome álcool ao menos uma vez ao mês (qui-quadrado, $p<0,006$; OR 3,6; IC $95 \%=1,3$ a 9,8), bem como esta relação significante surge na população que consomeálcool ao menos uma vez ao mês e declara que não esteve internada no último ano (qui-quadrado, $p$ $<0,013$; OR 1,8; IC $95 \%=1,1$ a 2,9).

Os níveis de depressão medidos pelo Inventário de Beck para Depressão (BDI) mostraram menores índices de depressão para aqueles que consomem álcool ao menos uma vez ao mês (quiquadrado, $p<0,047$; OR 2,0; IC $95 \%=0,99$ a 4,2). N ão seobservou correlação significanteentre o consumo regular de álcool no último mês (em quantidade de doses consumidas/dia deuso) e o nível de depressão geral (BDI- escalar). Também não se observou associação entre consumidores regulares de álcool e o grau de ansiedade mensurados pelo BAI, bem como não se observou correlação significante entre o consumo de álcool em quantidade de doses consumidas por dia de uso para os consumidores regulares e 0 nível da ansiedade (BAI - escalar). As variáveis estudadas podem ser observadas na Tabela 2.

\section{Discussão}

A OMS enfatiza que o número de usuários de álcool continua crescendo no Brasil e que, por existirem poucos estudos que evidenciem as diferenças regionais tais como as características econômicas, 0 acesso a educação e ao sistema de saúde, bem como as diferenças culturais existentes em sua extensa região geográfica, faz com que o país opte por programas de prevenção "importados" e que não se adéquam à realidade nacional ${ }^{10}$. Este estudo traz uma amplitude de informações acerca tanto da prevalência de consumidores regulares de determinada região do país, quanto do perfil desteusuário, nos aspectos questionados pela OMS.

Ainda segundo a OM S, o Brasil apresenta uma atitude extremamente tolerante em relação ao consumo de álcool e com mínimas restrições inclusive ao consumo evenda para menores de dezoito anos ${ }^{10}$. Reis et al. ${ }^{11}$ demonstraram que $10 \%$ das entradas por trauma em hospital brasileiro apresentavam intoxicação por álcool. Ainda, na contramão, e no que se refere ao controle das bebidas alcoólicas, estudos têm demonstrado 0 papel da propaganda que, além de pobremente regulada, incentiva o uso não apenas no clima social, mas também sobre o próprio comportamento deconsumo precoce ${ }^{12}$. Esteestudo não teve por objetivo observar a prevalência de consumo de álcool entre menores de idade ou no trauma; contudo, verificou que existe uma maior prevalência de consumo mensal entre pessoas mais jovens e predominantemente do sexo masculino. $\mathrm{N}$ a região sul do Brasil, Primo e Stei $\mathrm{n}^{13}$ também observaram que existemaior prevalência deabuso deálcool para o sexo masculino. Entre estudantes universitários na região nordeste do Brasil, Lemos et al. ${ }^{14}$ verificaram que o álcool já foi usado por $92,8 \%$ na vida e com prevalência maior também em homens. Na Região Sudeste, jovens ingressos em universidade mostram uma prevalência de consumo de álcool de $73,5 \%{ }^{15}$. Estes estudos indicam um consumo de álcool predominantemente em homens em diferentes regiões do Brasil.

O primeiro levantamento nacional sobre os padrões de consumo deálcool na população brasileira foi realizado entrenovembro de 2005 eabril de 2006 pela Secretaria Nacional Antidrogas (SE- 
Tabela 2. Comparação entre o consumo regular de álcool e sua relação com as variáveis independentes estudadas.

\begin{tabular}{|c|c|c|c|c|c|}
\hline \multirow[t]{2}{*}{ Variáveis } & \multicolumn{3}{|c|}{ Consumo regular de álcool } & \multirow{2}{*}{$\begin{array}{l}\text { Qui-quadrado } \\
\text { (IC 95\%) }\end{array}$} & \multirow{2}{*}{ Odds Ratio ${ }_{\text {Bruto }}$} \\
\hline & Sim & Não & Total & & \\
\hline \multicolumn{6}{|l|}{ Sexo } \\
\hline M asculino & 188 & 95 & 283 & \multirow[t]{2}{*}{$P<0,001$} & $4,2(3,1 a 5,9)$ \\
\hline Feminino & 134 & 290 & 424 & & 0,2 \\
\hline \multicolumn{6}{|l|}{ Idade } \\
\hline 18 a 39 anos & 184 & 181 & 365 & \multirow[t]{2}{*}{$P=0,007$} & $1,5(1,1 \mathrm{a} 2,0)$ \\
\hline Acima de 39 anos & 138 & 204 & 342 & & 0,6 \\
\hline \multicolumn{6}{|l|}{ Escolaridade (anos de estudo) } \\
\hline Até 8 & 78 & 150 & 228 & \multirow[t]{2}{*}{$P<0,001$} & 0,5 \\
\hline M ais de 8 & 238 & 230 & 468 & & $2,0(1,4$ a 2,7$)$ \\
\hline \multicolumn{6}{|l|}{ Renda familiar (mediana) } \\
\hline Até $\mathrm{R} \$ 1738,00$ & 118 & 213 & 331 & \multirow[t]{2}{*}{$P<0,001$} & 0,6 \\
\hline M ais de $R \$ 1738,00$ & 179 & 149 & 328 & & $2,1(1,5$ a 2,9$)$ \\
\hline \multicolumn{6}{|l|}{ Estado conjugal } \\
\hline Com companheiro(a) & 205 & 261 & 466 & NS & $*$ \\
\hline Sem companheiro(a) & 117 & 124 & 241 & NS & $*$ \\
\hline \multicolumn{6}{|l|}{ Situação ocupacional atual } \\
\hline Trabalha & 235 & 228 & 463 & \multirow[t]{2}{*}{$P<0,001$} & $1,8(1,3$ a 2,5$)$ \\
\hline Não trabalha & 87 & 157 & 244 & & 0,5 \\
\hline \multicolumn{6}{|c|}{ Como considera seu estado de saúde } \\
\hline M uito ruim/ruim & 5 & 21 & 26 & \multirow[t]{2}{*}{$P=0,006$} & 0,27 \\
\hline Regular/ muito bom & 317 & 364 & 681 & & $3,6(1,3$ a 9,8$)$ \\
\hline \multicolumn{6}{|c|}{ Esteve internado nos últimos 12 meses } \\
\hline Não & 294 & 328 & 622 & \multirow[t]{2}{*}{$P=0,013$} & $1,8(1,1$ a 2,9$)$ \\
\hline Sim & 28 & 57 & 85 & & 0,5 \\
\hline \multicolumn{6}{|c|}{$\begin{array}{l}\text { Níveis de ansiedade - Inventário Beck } \\
\text { de Ansi edade (BAI) }\end{array}$} \\
\hline M ínimo/Leve ( 0 a 16) & 228 & 281 & 509 & NS & $*$ \\
\hline M oderado/Grave (17 a 63) & 94 & 104 & 198 & NS & $*$ \\
\hline \multicolumn{6}{|c|}{$\begin{array}{l}\text { Níveis de depressão - Inventário de } \\
\text { Beck para Depressão (BDI) }\end{array}$} \\
\hline M ínimo/Leve ( 0 a 19) & 311 & 359 & 670 & \multirow[t]{2}{*}{$P=0,047$} & $2,0(0,1$ a 4,2$)$ \\
\hline M oderado/Grave (20 a 63) & 11 & 26 & 37 & & 0,48 \\
\hline
\end{tabular}

NS: Não significante; *Cálculo não realizado.

NAD ) ${ }^{16}$. Estelevantamento entrevistou 3.007 pessoas, sendo 2.346 adultas com mais de dezoito anos e 661 adolescentes entre catorze e dezessete anos em 143 municípios brasileiros, denortea sul do país. A pesquisa nacional aponta para uma diferença marcante com relação ao consumo de álcool entre homens e mulheres, que bebem com menor frequência e em menor quantidade. Em consideração à faixa etária, na faixa etária de 18 a 34 anos encontram-se os que consomem álcool com maior frequência, de forma semelhante aos resultados deste estudo. Os dados nacionais, para a Região Sul, mostram que maior porcentagem de pessoas que bebem está nas classes com maior renda, resultados estes também comparáveis aos encontrados em Joaçaba. Os dados levantados pelo SEN $A D^{16}$ mostram uma discrepância em relação aos resultados encontrados no presente estudo quanto à quantidade de consumidores de álcool classificados como "bebedor menos frequente" ou os que bebem entre uma a três vezes por mês, que na amostra nacional representou $17 \%$ comparado com $80,4 \%$ que declararam ter consumido álcool no último mês no presente estudo (Tabela 1). Por fim, a pesquisa nacional constatou que $48 \%$ da população adulta é abstinente ao álcool, enquanto que os dados revelados pela presente pesquisa indicam uma proporção de 
54,5\% deabstinência, evidenciando percentagens bastante próximas.

A relação entre álcool e a situação ocupacional relativa ao trabal ho tem sido pouco estudada. Em uma pesquisa realizada na Região Centro-O este do país, observou-se uma maior pre valência deuso recentedeálcool entreestudantes adolescentes que trabalhavam $(47,4 \%)$ do que os que não trabalhavam $(32,1 \%)^{17}$. Estes resultados se assemelham aos encontrados neste estudo com relação ao trabalho ea maior frequência de consumo de álcool; contudo, deve ser observada a diferença entre as populações no que concerne à faixa etária. Esta relação entre trabaIho e consumo deál cool não está clara enecessita demaior investigação no que diz respeito ao maior consumo na população economicamente ativa.

Tem sido demonstrado que o etanol influencia a liberação dos principais neurotransmissores presentes no SNC, tais como a dopamina, a serotonina, a noradrenalina e opióides ${ }^{18}$. Tais substâncias estão relacionadas à fisiopatologia da de pressão e desta forma o álcool pode estar influenciando os níveis de depressão indicados pelo BDI e evidenciados nesta pesquisa. O'D onnell et al. ${ }^{19}$ avaliaram a relação entre sintomas de depressão e o consumo de álcool em 15.748 pacientes de vinte países e observaram maiores chances de es- cores el evados do BDI para os que declaram não beber $(1,22$; IC 1,06-1,42) em relação aos que bebem, de forma semel hante aos resultados de J oaçaba. Por outro lado, Bravo de Medina et al. ${ }^{20}$ observaram que, para aqueles com dependência ao álcool e que buscaram ajuda terapêutica, os índices do BDI foram superiores ao grupo controle composto por pacientes saudáveis.

\section{Conclusões}

Este estudo demonstrou que aproximadamente $45 \%$ da população adulta de J oaçaba (SC) consome álcool regularmente e esta população caracteriza-se por estar trabal hando, ser jovem e do sexo masculino, independente do estado conjugal, ter uma renda acima da mediana encontrada (1.738,00 reais) e ter escolaridade superior a oito anos. Ainda, este consumo regular de álcool de ao menos uma vez ao mês mostrou-se mais frequentemente associado naqueles que declararam considerar sua saúde como regular a muito boa e, também, observou-se que entre estes consumidores regulares existem menores índices de depressão (BDI) quando comparados com a população que declara não consumir álcool regularmente.

\section{Colaboradores}

MC Bortoluzzi, J Traebert, A Loguercio e RT Kehrig participaram igualmente de todas as etapas da elaboração do artigo. 


\section{Referências}

1. World Health Organization. WHO Library Cataloguing-in-Publication Data. Global status report on alcohol 2004. [site da Internet]. [acessado $2008 \mathrm{fev].}$ Disponível em: http://www.who.int/substance_ abuse/publications/alcohol/en/index.html

2. World Health Organization. WHO Library Cataloguing-in-Publication Data. Alcohol and injury in emergency departments: summary of the report from the WHO collaborative study on alcohol and injuries (2007). [site da Internet]. [acessado 2008 fev]. Disponível em: http://www.who.int/substance abuse/ publications/alcohol/en/index.html

3. World Health Organization. WHO Library Cataloguing-in-Publication Data. Alcohol in developing societies: a public health approach. Summary (2002). [site da Internet]. [acessado 2008 fev]. Disponível em: http://www.who.int/substance_abuse/publications/alcohol/en/index.html

4. Lakins NE, LaVallee RA, Williams GD, Yi H. National Institute on Alcohol Abuse and Alcoholism Division of Epidemiology and Prevention Research Alcohol Epidemiologic Data System. Apparent per Capita Alcohol Consumption: National, State, and Regional Trends, 1977-2005. Surveillance Report \#82 (2007). [site da Internet]. [acessado $2008 \mathrm{fev}$ ]. Disponível em: http://pubs.niaaa.nih.gov/publications/surveillance.htm

5. Brasil. Ministério da Saúde. Secretaria Executiva. Coordenação Nacional de DST/Aids. A Política do M inistério da Saúde para atenção integral a usuários de álcool e outras drogas. - Brasília: Ministério da Saúde; 2003.

6. Kehrig RT, Traebert J. Auto-referência de problemas de saúde, condições de vida e respostas dos serviços para a população adulta (Joaçaba, 2005) [projeto de pesquisa CNPQ Processo 402829/05-0]. Brasília: CNPQ; 2005.

7. Pizzatto M, Silva RM, Traebert J. Cefaléia associa$\mathrm{da}$ a indicadores de transtornos de ansiedade em uma população adulta do sul do Brasil: um estudo de base populacional. Revista da Sociedade Brasileira de Clínica M édica 2008; 6:15-20.

8. Medronho RA. Epidemiologia. São Paulo: Atheneu; 2002.

9. Cunha JA. M anual da versão em português das Escalas Beck. São Paulo: Casa do Psicólogo; 2001.

10. World Health Organization. WHO Library Cataloguing-in-Publication Data. Alcohol, gender and drinking problems: perspectives from low and middle income countries (2005). [site da Internet]. [acessado $2008 \mathrm{fev}$ ]. Disponível em: http://www.who.int/ substance_abuse/publications/alcohol/en/index. $\mathrm{html}$
11. Reis AD, Figlie NB, Laranjeira R. Prevalence of substance use among trauma patients treated in a Brazilian emergency room. Rev. Bras. Psiquiatr. 2006; 28(3):191-195.

12. Pinsky I, Pavarino Filho RV. A apologia do consumo de bebidas alcoólicas e da velocidade no trânsito no Brasil: considerações sobre a propaganda de dois problemas de saúde pública. Rev. psiquiatr. Rio Gd. Sul. 2007; 29(1):110-118.

13. Primo NLNP, Stein AT. Prevalência do abuso e da dependência de álcool em Rio Grande (RS): um estudo transversal de base populacional. Rev. psiquiatr. Rio Gd. Sul. 2004; 26(3):280-286.

14. Lemos KM, Neves NM B, Kuwano AY, Tedesqui G, Bitencourt AGV, Neves FBCS, Serra FBC, Guimarães NA, Rebello A, Bacellar F, Lima MM. Uso de substâncias psicoativas entre estudantes de M edicina de Salvador (BA). Rev. psiquiatr. clín. 2007; 34(3):118-124.

15. Vieira VCR, Riore SE, Ribeiro SMR, Franceschini SCC, Almeida LP. Perfil socioeconômico, nutricional e de saúde de adolescentes recém-ingressos em uma universidade pública brasileira. Rev. Nutr. 2002; 15(3):273-282.

16. Laranjeira R, Pinsky I, Zaleski M, Caetano R. I Levantamento $\mathrm{N}$ acional sobre os padrões de consumo de álcool na população brasileira. [site da Internet]. [acessado 2008 out]. Disponível em: http:// www.obid.senad.gov.br

17. Souza DPO, Silveira Filho DX. Uso recente de álcool, tabaco e outras drogas entre estudantes adolescentes trabalhadores e não trabalhadores. Rev. bras. epidemiol. 2007; 10(2):276-287.

18. Zaleski M, M orato GS, Silva VA, Lemos T. Aspectos neurofarmacológicos do uso crônico e da Síndrome de Abstinência do Álcool. Rev. Bras. Psiquiatr. 2004; 26(Suppl.1):40-42.

19. O'Donnell K, Wardle J, Dantzer C, Steptoe A. Alcohol consumption and symptoms of depression in young adults from 20 countries. J Stud Alcohol. 2006; 67(6):837-840.

20. Bravo de Medina R, Echeburúa E, Aizpiri J. Psychopathological symptoms and personality traits in alcohol-dependent patients: a comparative study. Adicciones 2007; 19(4):373-381.

Artigo apresentado em 19/05/2008

Aprovado em 02/10/2008

Versão final apresentada em 29/10/2008 